\title{
Cholecystectomy and the Risk of Colorectal Adenomas
}

\author{
Lisa C. Vinikoor ${ }^{1}$, Joseph A. Galanko ${ }^{2}$, and Robert S. Sandler ${ }^{2}$ \\ 1Department of Epidemiology, School of Public Health, School of Medicine, University of North Carolina, \\ Chapel Hill, North Carolina \\ 2Department of Medicine and Center for Gastrointestinal Biology and Disease, School of Medicine, \\ University of North Carolina, Chapel Hill, North Carolina
}

\begin{abstract}
Background-Cholecystectomy has been identified as a risk factor for colorectal cancer, yet little attention has been given to the association between cholecystectomy and colorectal adenomas.

Methods-We utilized data collected in two large cross-sectional studies of colorectal adenoma risk factors to examine the association between cholecystectomy and colorectal adenomas. Consecutive patients undergoing colonoscopies at the University of North Carolina Hospitals were recruited into the studies. Diet and lifestyle questionnaires were given to participants within twelve weeks of their colonoscopy. Logistic regression was used to estimate the prevalence odds ratios overall, by site of adenoma, and by time since cholecystectomy.
\end{abstract}

Results-In the adjusted logistic regression model, both men and women showed no effect of cholecystectomy on risk of colorectal adenomas. The point estimates for men and women were in opposite directions (men: odds ratio (OR) 0.67 ; $95 \%$ confidence interval (CI) 0.30, 1.47; women: OR $1.46 ; 95 \%$ CI $0.92,2.29)$. No effect was seen when examining the time since cholecystectomy for men (cholecystectomy at least ten years prior to entering the study: OR $0.94 ; 95 \%$ CI $0.28,3.22$; cholecystectomy less than ten years prior: OR $0.53 ; 95 \%$ CI $0.19,1.50$ ) or women (cholecystectomy at least ten years prior 1.14 [ $95 \%$ CI $0.62,2.09$ ], cholecystectomy less than ten years prior 2.02 [95\% CI 1.06, 3.87]).

Conclusion-We conclude that there is no simple relationship between factors that result from cholecystectomy, such as exposure of the colon to bile salts, and colorectal adenoma risk.

\section{Keywords}

Epidemiology; Risk Factor; Colorectal Adenoma; Cholecystectomy

\section{INTRODUCTION}

Cholecystectomy remains one of the most commonly performed operations in the United States. The rates of cholecystectomy have fluctuated in the United States but have remained consistently above 150 per 100,000 people per year since the 1990s (1). With this number of cholecystectomies being performed, it is important to understand the potential health risks associated with this procedure. One outcome that has not been thoroughly addressed is the possibility of an association between cholecystectomy and colorectal adenomas.

\section{Correspondence to: Lisa C. Vinikoor.}

Requests for reprints: Lisa C. Vinikoor, Department of Epidemiology/CGIBD, CB\#7555, University of North Carolina, Chapel Hill, NC 27599-7555. Phone: 919-966-0090; Fax: 919-966-2478; E-mail: vinikoor@email.unc.edu.. 
Many studies have examined the association between cholecystectomy and colorectal cancer. Two meta-analyses have reported an increased risk of colorectal cancer in those who have undergone a cholecystectomy $(2,3)$. Some, but not all, of the studies used in the two metaanalyses overlapped. Both meta-analyses found that the risk of colorectal cancer was higher in the right side of the colon. They also demonstrated a higher risk in women. Since the publication of the meta-analyses, more studies have been published looking at this association. Some found an increase in the risk of proximal colon tumors $(3,4)$. One study reported an increase in sigmoid tumors for women that had undergone a cholecystectomy (6). A study performed only in women found that there was an increase in cancer associated with cholecystectomy, and more specifically, an increase in the risk of proximal colon and rectal cancer (7). Other studies showed an increased risk of colon cancer after cholecystectomy but no increase for rectal cancer (8) or no association at all between cholecystectomy and advanced colonic neoplasia (9).

While there have been a number of studies evaluating the association between cholecystectomy and cancer, few have studied the association between cholecystectomy and colorectal adenomas. The meta-analyses performed by Giovannucci et al. analyzed a subset of papers reporting on colorectal adenoma risk as well colorectal cancer risk (3). Many of the studies lacked an adequate sample size to investigate the association between cholecystectomy and increased risk of colorectal adenomas, and few studies controlled for potential confounders, particularly potentially important confounders such as diet $(2,3)$. Failure to account for an induction period may also have affected the findings (3).

Two possible mechanisms have been suggested for the relationship between cholecystectomy and colorectal neoplasia. First, cholecystectomy might operate to speed the transition of adenomas to cancer. Alternatively, cholecystectomy might increase the risk for adenomas, thereby creating more opportunities for the development of colorectal cancer. Determining which of the above two possible mechanisms is correct will not only provide etiologic information for understanding of the development of colorectal cancer but could potentially have policy and procedural implications. For example, if the increased risk is related to the progression of adenomas into cancer, patients (and their doctors) might take more aggressive measures in terms of screening. In addition, those at higher risk might elect to further modify their lifestyle to lower their risk. In order to further clarify the association between cholecystectomy and colorectal adenomas, we conducted a hospital-based cross-sectional study of cholecystectomy and adenomas that collected information on possible confounders, effect measure modifiers, location of the adenomas in the colon, and time interval between cholecystectomy and adenoma development.

\section{METHODS}

\section{Study Population}

The Diet and Health Study III (DHS III) and the Diet and Health Study IV (DHS IV) recruited consecutive patients undergoing an outpatient colonoscopy at the University of North Carolina Hospitals in Chapel Hill, North Carolina from August 1998 to March 2000 (DHS III) and from November 2001 to December 2002 (DHS IV). Eligible patients were between the ages of 30 and 80. Patients were excluded if they did not speak English, or had familial polyposis, colitis, previous colonic resection, or previous colon cancer or adenoma. Eligible patients were asked to consent to a telephone-administered interview and to a rectal biopsy of normal mucosa collected during the colonoscopy. 


\section{Data Collection}

There were 5621 colonoscopies performed at the University of North Carolina Hospitals during the study period. Of these, 1677 (30\%) were eligible to participate in the study. Exclusions were due to: age $(n=464)$, familial polyposis $(n=10)$, colitis $(n=710)$, previous colonic resection $(n=518)$, previous colon cancer or adenoma $(n=1224)$, unsatisfactory prep $(n=250)$, and incomplete exam $(n=239)$. These reasons are not mutually exclusive. Of the 1677 eligible patients (926 from DHS III and 751 from DHS IV), 1437 (86\%) consented to be a part of the study and completed the telephone interview. The primary indication for colonoscopy was screening for the vast majority of participants.

A telephone interview was conducted within twelve weeks of the colonoscopy. Patients were asked questions about their lifestyle, including physical activity (asking both amount of time spent doing activities and how strenuous these activities were, which were later converted to metabolic equivalents per day), use of non-steroidal anti-inflammatory drugs (NSAIDs) (asking how many times per day or week a person used NSAIDs), and medical conditions/ operations. They were also given a detailed dietary questionnaire. The participants of DHS III were given a modified version of the Block Food Frequency Questionnaire $(10,11)$.

Modification to the questionnaire was made in order to include foods more common in North Carolina, such as hush puppies and black-eyed peas. Subjects in DHS IV were given the NCI Diet History Questionnaire (12). All subjects were asked to report their diet over the past year (allowing for seasonal fluctuations in patterns of consumption). Participants were questioned about dietary supplements as well.

Since two different dietary questionnaires were used, the values for dietary variables between DHS III and DHS IV could not be directly combined. Instead, the variables were divided into quartiles based on the controls for each study and then combined using their assigned quartile resulting in categorical dietary variables for each participant.

\section{Data Analysis}

Cases were defined as participants who had a colorectal adenoma detected. Location and size of the adenoma were also noted. Participants with no adenomas detected were defined as controls. If a person received a diagnosis of colorectal cancer, they were excluded from the analysis.

Information on cholecystectomy was collected during the telephone interview. Participants were asked if they ever had a cholecystectomy, and, if so, how long ago they underwent this procedure.

Missing exposure data was assessed in order to determine if information was missing differentially by outcome. Information about past cholecystectomies was missing for $7.29 \%$ of the cases and $7.11 \%$ of the controls. While it does not appear that missing exposure data was affected by outcome, those missing this information were excluded from the analysis.

Logistic regression was used to model the data. Prevalence odds ratios and 95\% confidence intervals were calculated using STATA/SE (STATA Corp. 2005. Statistical Software: version 8.2. College Station, TX). In the main analysis, case-control status was used as the outcome variable and prior cholecystectomy was the exposure variable. The following variables were assessed for effect measure modification and for confounding: age (coded as a continuous variable), race (coded as white and non-white), sex (coded as a binary variable), smoking (coded as an indicator variable with three categories: never, former, and current smoker), body mass index (BMI) (coded as a continuous variable), physical activity (coded continuously using current met-minutes per day), NSAID use (coded continuously as number of pills per month), and dietary variables measuring calories, fat, carbohydrates, and fiber consumed (each of these 
was coded categorically in quartiles using the afore mentioned methods). Examination for effect measure modification was performed using the Breslow-Day Test of Homogeneity. A p-value of 0.15 was used as the cut-off value for classifying a variable as an effect measure modifier. If a variable was determined to be an effect measure modifier, it was entered into the final model along with an interaction term. Prevalence odds ratios and $95 \%$ confidence intervals were calculated within each stratum of this variable. Linearity of the logit was also assessed in order to verify the linearity assumption of logistic modeling. All of the variables satisfied this assumption and thus no additional indicator variables were used. Confounding by the variables of interest was assessed using the change-inestimate approach through backward elimination. The change-in-estimate between the "full" model and the model without the variable of interest was calculated. If the change was greater than $10 \%$ then the variable was considered to be a confounder. In order to avoid deleting observations with missing information on certain covariates, multiple imputation was performed. A regression was run using the missing variable as the dependent variable. The independent variables were those that might allow us to discern the value of this missing variable. The regression was run multiple times and the results were combined to form a single estimate.

A secondary analysis was undertaken to examine if the risk of colorectal adenomas was greater on a specific side of the colon. Prevalence odds ratios and 95\% confidence intervals within the strata of any previously determined effect measure modifier were calculated for the left and right sides of the colon using the controls as the common referent. Adjustments were made for any variables already determined to be confounders. The right side of the colon was defined as the cecum, ascending colon, hepatic flexure, and transverse colon. The left side of the colon was considered the splenic flexure, descending colon, sigmoid colon, and rectum. If a person had an adenoma detected on both the left and right sides of their colon they were excluded from this analysis.

Another analysis was performed to examine the possible change in risk with time since undergoing a cholecystectomy. This was done by categorizing participants based on the amount of time that had passed since their cholecystectomy. Prior studies have used a cut-point of ten years $(13,14)$. Therefore, we categorized people as never having a cholecystectomy, having had a cholecystectomy performed less than 10 years before their enrollment in the study, and having undergone a cholecystectomy ten years or more before becoming part of the study. Then, the prevalence odds ratios and $95 \%$ confidence intervals were calculated with adjustment for any previously determined confounders and within the stratum of any effect measure modifiers using the group that never had a cholecystectomy as the referent population.

\section{RESULTS}

A total of 1,437 patients were included in the final study population. Of these, 425 were cases (29.58\%) and 1,012 were controls (70.42\%). One hundred fifty-one participants had undergone a cholecystectomy (11.32\%). There were more women than men, with women comprising approximately $57 \%$ of the study population. The mean age was 56 years. Table 1 gives the characteristics of the study population by case and control status. Cases were older than controls and had slightly higher BMIs. Cases also included more men than women, whereas controls had a higher proportion of women than men. Women in the study population were slightly older than the men, and a higher percentage of women than men had undergone a cholecystectomy.

The only effect measure modifier among the variables of interest was sex. The change-inestimate approach yielded six confounders. All variables found to be confounders using the change-in-estimate approach were adjusted for in the analyses. The confounders of the 
prevalence odds ratio were calories (categorical), age (continuous), race (categorical), BMI (continuous), NSAID use (continuous), and daily physical activity (continuous).

When controlling for these confounders the prevalence odds ratios (POR) and their respective 95\% confidence intervals were 0.67 (95\% CI: 0.30, 1.47) for men and 1.46 (95\% CI: 0.92, 2.29) for women (Table 2). While the $95 \%$ confidence intervals for men and women included the null values, the prevalence odds ratios were in different directions. The adjusted estimates suggest that the risk of colorectal adenomas is decreased for men who underwent cholecystectomy. The risk of colorectal adenomas was increased in women. When considering not only the point estimates but the $95 \%$ confidence intervals as well, our study shows no effect of cholecystectomy on the risk of colorectal adenomas.

We attempted to determine whether risk increased for adenomas of the left or right side of the colon. Of the 425 cases in the study, 1 was missing information on location, 148 had adenomas of the left colon, 225 had adenomas of the right colon, and 51 had adenomas on both the left and right colon. There were not enough men to support analysis. Only 2 men in the study population who had undergone a cholecystectomy had an adenoma detected only on the left side of their colon and only to 6 men had solely right-sided adenomas. Women, on the other hand, had adequate numbers to conduct the analysis but no increase in risk for either side was seen. When compared to controls, the prevalence odds ratio (and 95\% confidence interval) for women having an adenoma detected on the left side of the colon was 1.37 (95\% CI: 0.70, 2.67). The prevalence odds ratio (and 95\% confidence interval) for right-sided adenomas was 1.44 (95\% CI: 0.78, 2.66).

In the ten years prior to entering the study, 70 people had cholecystectomies whereas 76 people had undergone a cholecystectomy at least ten years before their enrollment in the study. Men showed a decrease in the protective effect of cholecystectomy with increased time since cholecystectomy; however both odds ratios include the null and potentially show no effect of cholecystectomy on the risk of colorectal adenomas (Table 3). For women, there appears to be a slight increase in risk with cholecystectomy less than ten years prior (Table 3 ). This risk decreases towards the null when examining women who had a cholecystectomy at least ten years before entering the study. Of importance, however, is the mean time since cholecystectomy of study participants (Table 4). For women the median number of years since cholecystectomy was 11.0 years, with a mean of 13.9 years (SE 11.6 years). The median and mean number of years since their cholecystectomy for men was 5.5 years and 8.8 years (SE 9.5), respectively. The differences between the mean times since cholecystectomy were not statistically significant when comparing cases and controls for men $(\mathrm{p}=0.29)$ or women $(\mathrm{p}=0.39)$. The difference in the mean time since cholecystectomy for women and men was 5.1 years (p-value of 0.0130), showing that the men in our study had a much shorter period of time between cholecystectomy and colonoscopy when compared with the women in our study.

\section{DISCUSSION}

When controlling for confounders, this study found that cholecystectomy reduced the risk of developing colorectal adenomas for men. The risk was reduced more than $30 \%$ in men who underwent a cholecystectomy compared with men who did not. The $95 \%$ confidence interval for this odds ratio, as well as the confidence intervals for the time since cholecystectomy analysis are wide and include the null. A protective effect has not been demonstrated in past studies, but very few studies have had adequate samples of men. Prior studies with sizeable numbers of men have shown no effect of cholecystectomy in men $(13,15-18)$. While our study showed a protective affect when examining the point estimate, the confidence intervals show no effect of cholecystectomy. 
In contrast, we found an increased risk of colorectal adenomas for women but this also was not statistically significant. The same was found in sub-analysis of both the side of the colon and the time since cholecystectomy. The effect seen in women is similar to other studies that found null values after adjusting for confounders $(16,17)$. Other studies that have found an increased risk in women had either small sample size or found an increase only in the rectum $(7,13,15,18)$

When looking at both sexes, the time since cholecystectomy affects the magnitude of the risk. For men, cholecystectomy becomes less protective when men have had a cholecystectomy ten or more years prior. For women, the elevated risk decreases. Though the point estimate of the odds ratio for both men and women moves closer to the null, the confidence intervals are too wide to estimate a change of risk with time. Two prior studies found an increased risk colorectal adenomas with time since cholecystectomy, however these were performed in people 60 years of age and older who had a cholecystectomy performed at least ten years prior $(13,14)$. The study population used in our analysis was not large enough to assess that age group alone. We did however control for age in our analysis.

It has been proposed that removing the gallbladder exposes the intestines to a constant lowlevel flow of bile, which may modify the environment of the colon $(3,4)$. If bile salts were the sole mechanism one would expect to observe similar effects in men and women, which is not the case. Also, increasing time does not have an effect on risk. Thus, longer exposure to bile is not increasing the risk of colorectal adenomas. Because there appears to be an increased risk of colorectal cancer following cholecystectomy $(2,3)$, and because our study has shown that there is no increase in risk for colorectal adenomas, the effects of cholecystectomy on risk of colorectal cancer must occur later in the neoplastic process, after the development of the adenoma. The observed difference in risk when adenomas are the outcome measure, as opposed to cancer as the outcome illustrates that different risk factors may occur at different points in the adenoma-carcinoma sequence. Furthermore, the observation illustrates the hazards of using intermediate markers, such as adenomas, as endpoints, as they might falsely reject a risk for cancer. However, caution must be taken not to remove people with adenomas from the control group in case-control studies of cholecystectomy and colorectal cancer. Doing this not only has the potential to bias the results but the trohoc fallacy is also committed (19).

While this study contributes to the knowledge of cholecystectomy and risk of colorectal adenomas, it has limitations. First, the sample size is small, limiting further analysis and decreasing precision. Also, the information on cholecystectomy was missing for approximately $7 \%$ of the study participants. Finally, dietary practices are assumed to be relatively constant throughout life. If, however, this is not the case, then information on diet ten or so years prior may have been more informative than the diet recorded and studied in DHS III and DHS IV.

In sum, the present study suggests that cholecystectomy affects the risk of colorectal adenomas differently for men and women. There is no difference seen for the length of time after a cholecystectomy. Future studies of cholecystectomy and adenoma risk should examine men and women separately. Work should be done to look at biologic factors besides bile salts that may create an increased risk in adenoma becoming cancerous.

\section{ACKNOWLEDGEMENTS}

We would like to thank Dr. Alexandra C. Frost for providing invaluable background information on DHS III and DHS IV and Dr. David Ransohoff for his reviews of this manuscript.

Grant support: Supported in part by grants from the NIH Grants T32-DK07634, R01 DK 44684, and P30 DK34987. 


\section{REFERNCES}

1. Urbach DR, Swantstrom LL, Khajanchee YS, et al. Incidence of cancer of the pancreas, extrahepatic bile duct and ampulla of Vater in the United States, before and after the introduction of laparoscopic cholecystectomy. Am J Surg 2001;181:526-8. [PubMed: 11513778]

2. Reid FDA, Mercer PM, Harrison M, et al. Cholecystectomy as a risk factor for colorectal cancer: a meta-analysis. Scand J Gastroenterol 1996;31:160-9. [PubMed: 8658039]

3. Giovannucci E, Colditz GA, Stampfer MJ. A meta-analysis of cholecystectomy and risk of colorectal cancer. Gastroenterology 1993;105:130-41. [PubMed: 8514030]

4. Lagergren J, Weimin Y, Ekbom A. Intestinal cancer after cholecystectomy: Is bile involved in carcinogenesis? Gastroenterology 2001;121:542-7. [PubMed: 11522737]

5. Todoroki I, Friedman GD, Slattery ML, et al. Cholecystectomy and the risk of colon cancer. Am J Gastroenterol 1999;94:41-6. [PubMed: 9934729]

6. Neagoe A, Molnar AM, Acalovschi M, et al. Risk factors for colorectal cancer: an epidemiologic descriptive study of a series of 333 patients. Rom J Gastroenterol 2004;13:187-93. [PubMed: 15470530]

7. Schernhammer ES, Leitzmann MF, Michaud DS, et al. Cholecystectomy and the risk for developing colorectal cancer and distal colorectal adenomas. Br J Cancer 2003;88:79-83. [PubMed: 12556963]

8. Shao T, Yang YX. Cholecystectomy and the risk of colorectal cancer. Am J Gastroenterol 2005;100:1813-20. [PubMed: 16086719]

9. Lieberman DA, Prindiville S, Weiss DG, et al. Risk factors for advanced colonic neoplasia and hyperplastic polyps in asymptomatic individuals. Jama 2003;290:2959-67. [PubMed: 14665657]

10. Block G, Hartman AM, Naughton D. A reduced dietary questionnaire: development and validation. Epidemiology 1990;1:58-64. [PubMed: 2081241]

11. Block G, Hartman AM, Dresser CM, et al. A data-based approach to diet questionnaire design and testing. Am J Epidemiol 1986;124:453-69. [PubMed: 3740045]

12. Subar AF, Thompson FE, Kipnis V, et al. Comparative validation of the Block, Willett, and National Cancer Institute food frequency questionnaires: the eating at America's table study. Am J Epidemiol 2001;154:1089-99. [PubMed: 11744511]

13. Moorehead RJ, Mills JO, Wilson HK, et al. Cholecystectomy and the development of colorectal neoplasia: a prospective study. Ann R Coll Surg Engl 1989;71:37-9. [PubMed: 2923417]

14. Mannes AG, Weinzierl M, Stellaard F, et al. Adenomas of the large intestine after cholecystectomy. Gut 1984;25:863-6. [PubMed: 6745725]

15. Sandler RS, Martin ZZ, Carlton NM, et al. Adenomas of the large bowel after cholecystectomy. Dig Dis Sci 1988;33:1178-84. [PubMed: 3409804]

16. Neugut AI, Murray TI, Gabowski GC, et al. Cholecystectomy as a risk factor for colorectal adenomatous polyps and carcinoma. Cancer 1991;68:1644-7. [PubMed: 1893365]

17. Neugut AI, Johnsen CM, Forde KA, et al. Cholecystectomy and adenomatous polyps of the colon in women. Cancer 1988;61:618-21. [PubMed: 3338028]

18. Llamas KJ, Torlach LG, Ward M, et al. Cholecystectomy and adenomatous polyps of the large bowel. Gut 1986;27:1181-5. [PubMed: 3781331]

19. Poole C. Controls who experienced hypothetical causal intermediates should not be excluded from case-control studies. Am J Epidemiol 1999;150:547-551. [PubMed: 10489992] 
Table 1

Characteristics of cases and controls, Diet and Health Studies III and IV*

\begin{tabular}{|c|c|c|}
\hline & Colorectal Adenoma (cases) $\mathrm{N}=\mathbf{4 2 5}$ & $\begin{array}{l}\text { No Colorectal Adenoma (controls) } \\
\qquad \mathrm{N}=\mathbf{1 , 0 1 2}\end{array}$ \\
\hline Cholecystectomy (\%) & $45(11.42)$ & $106(11.28)$ \\
\hline Male Sex $(\%)$ & $240(56.47)$ & $373(36.86)$ \\
\hline White Race (\%) & $325(79.46)$ & $774(79.06)$ \\
\hline \multicolumn{3}{|l|}{ Smoking Status (\%) } \\
\hline Never & $180(46.04)$ & $476(50.64)$ \\
\hline Former & $148(37.85)$ & $320(34.04)$ \\
\hline Current smoker & $63(16.11)$ & $144(15.32)$ \\
\hline \multicolumn{3}{|l|}{ Age $(\%)$} \\
\hline$<40$ years & $11(2.70)$ & $68(7.03)$ \\
\hline $40-49$ years & $59(14.46)$ & $218(22.54)$ \\
\hline $50-59$ years & $163(39.95)$ & $369(38.16)$ \\
\hline $60-69$ years & $98(24.02)$ & $217(22.44)$ \\
\hline$\geq 70$ years & $77(18.87)$ & $95(9.82)$ \\
\hline Mean Age (SD) & $58.62(10.24)$ & $54.90(10.69)$ \\
\hline \multicolumn{3}{|c|}{ Body Mass Index (BMI) (\%) } \\
\hline$<25.0$ & $138(33.33)$ & $398(40.57)$ \\
\hline $25.0-29.9$ & $137(33.09)$ & $324(33.03)$ \\
\hline$\geq 30$ & $139(33.57)$ & $259(26.40)$ \\
\hline Mean BMI (SD) & $28.27(6.05)$ & $27.38(6.13)$ \\
\hline
\end{tabular}

* Calculated by using values prior to imputation 
Table 2

Association between colorectal adenomas and cholecystectomy by sex

\begin{tabular}{lcccc}
\hline & N Cases & N Controls & Prevalence Odds Ratio $^{*}$ & 95\% Confidence Interval $^{*}$ \\
\hline Men & 10 & 22 & 0.67 & $0.30,1.47$ \\
Cholecystectomy & 206 & 323 & 1.00 & $0.92,2.29$ \\
No cholecystectomy & 35 & 84 & 1.46 & 1.00 \\
Women & 143 & 511 & & \\
Cholecystectomy & 143 & & \\
No cholecystectomy & & & & \\
\hline
\end{tabular}

* Adjusted for age, BMI, NSAID use, calorie consumption, physical activity, and race 
Table 3

Association of colorectal adenomas and cholecystectomy by time since cholecystectomy and sex

\begin{tabular}{lcccc}
\hline & N Cases & N Controls & Prevalence Odds Ratio $^{*}$ & 95\% Confidence Interval $^{*}$ \\
\hline Men & 5 & 16 & 0.53 & $0.19,1.50$ \\
$<10$ years & 5 & 6 & 0.94 & $0.28,3.22$ \\
$\geq 10$ Years & 206 & 323 & 1.00 & $1.06,3.87$ \\
No cholecystectomy & 16 & 33 & 2.02 & $0.62,2.09$ \\
Women & 17 & 48 & 1.14 & 1.00 \\
$<10$ Years & 143 & 511 & & \\
$\geq 10$ Years & & & & \\
No cholecystectomy & & & & \\
\end{tabular}

* Adjusted for age, BMI, NSAID use, calorie consumption, physical activity, and race 


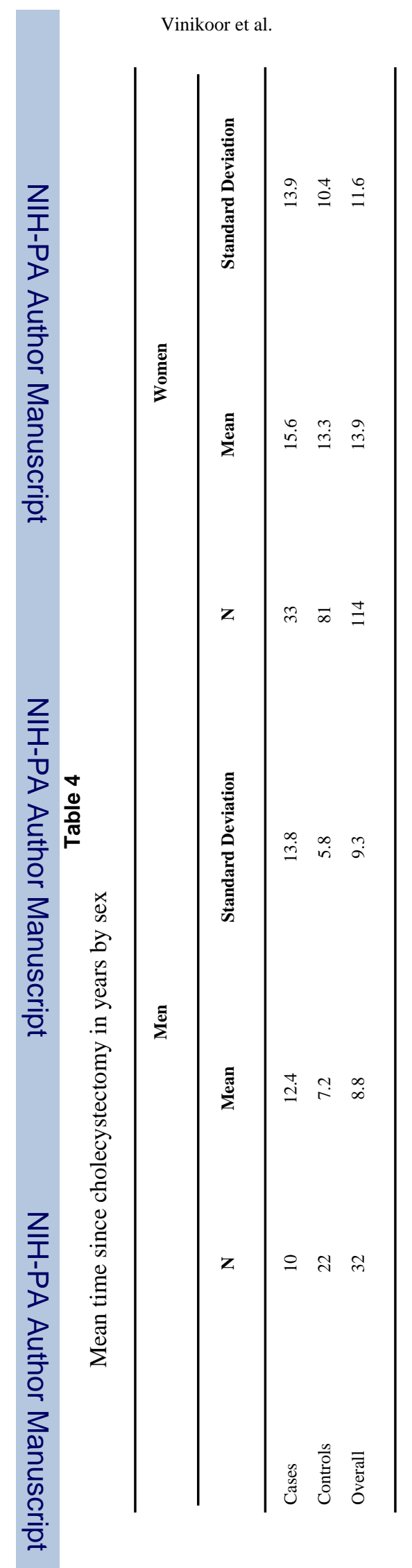

Dig Dis Sci. Author manuscript; available in PMC 2009 March 1. 